\title{
Conjugation of curcumin with Ag nanoparticle for improving its bioavailability and study of the bioimaging response
}

\author{
Runjun Sarma ${ }^{1 *}$, Monoj Kumar Das ${ }^{2}$, Lakshi Saikia $^{3}$, Anand Ramteke $^{2}$, Ratul Saikia ${ }^{4}$ \\ ${ }^{1}$ Department of Physics, Mehr Chand Mahajan DAV College for Women, Sector 36, Chandigarh, India \\ ${ }^{2}$ Cancer Genetics and Chemoprevention Research Group, Department of Molecular Biology and Biotechnology, \\ Tezpur University, Tezpur, Assam 784028, India \\ ${ }^{3}$ Material Science and Technology Division, CSIR-North East Institute of Science and Technology, \\ Jorhat, Assam 785006, India \\ ${ }^{4}$ Biological Science and Technology Division, CSIR-North East Institute of Science and Technology, \\ Jorhat, Assam 785006, India \\ *runjun2018chd@gmail.com
}

DOI 10.17586/2220-8054-2021-12-4-528-535

\begin{abstract}
We report here the production of curcumin conjugated polyvinylpyrrolidone (PVP)-capped Ag nanoparticles for potential biological applications. Evidence for the efficient conjugation of hydrophobic curcumin to the synthesized nanoparticles (NPs) is expected from UV-Vis, zeta potential and Fourier transform infrared (FT-IR) analysis. Curcumin conjugated to PVP-capped Ag NPs is observed to gain high water solubility and bioavailability in a biological environment without diminishing its therapeutic properties. The presence of the main therapeutic group, the diarylheptanoid chromophore of curcumin, indicates the existence of its medicinal behavior in the curcumin-Ag NPs complex. Moreover, the fluorescence efficiency of PVP capped Ag NPs in the breast cancer cellular medium have been found to be significantly enhanced (by factor of $\sim 2.37$ ) with curcumin conjugation. The production of bioavailable curcumin provides an opportunity to expand the clinical repertoire of this efficacious agent without hampering the environment and human health.
\end{abstract}

Keywords: therapeutic, bioavailability, imaging, curcumin, PVP.

Received: 15 March 2021

Revised: 22 July 2021

\section{Introduction}

Curcumin (Turmeric), a bio-active polyphenol component of Curcuma longa L., has attracted significant interest in biological research owing to its unique therapeutic properties such as anti-inflammatory, antibacterial, antioxidant, antifungal, anti-carcinogenic etc. [1,2]. Moreover, it has been found that curcumin is extremely safe even at very high doses in an animals as well as in humans [3,4]. Therefore, curcumin exhibits tremendous potential to be used for the treatment and prevention of various human diseases. However, despite the possibility for the use of curcumin as a therapeutic agent in vivo, poor aqueous solubility (i.e. $0.0004 \mathrm{mg} / \mathrm{mL}$ at $\mathrm{pH} 7.3$ ) limits its use as a drug for the prevention or treatment of various diseases. This results in low intrinsic activity, poor absorption and rapid elimination/clearance from the living body [1].

Until now, various methods have been reported to explore the potential applications of curcumin in various biological fields. Nanoparticles, liposomes, micelles, and phospholipid complexes have been used to improve permeability and circulation of curcumin in vivo. However, it has been observed that though the bio-availability improves upon conjugation, the main therapeutic group of this molecule gets engaged in the conjugation thereby decreasing its availability for therapeutic activity in a biological system [5]. In this regard, the conjugation of curcumin in the nanoparticle-polymer composite may be an alternative and easier approach to enhance its water solubility as well as bioavailability without diminishing its therapeutic property [6].

On the other hand, in recent years, metallic nanoparticles (NPs) in general and silver NPs (Ag NPs) in particular have attracted considerable interest in various applications such as therapeutic [7], drug delivery [8], antimicrobial [9] and medical imaging [10]. This is due to their unique physical, chemical, optical and biological properties [11].

We report here on the formation of water-soluble and biocompatible curcumin conjugated Ag NPs. Polyvinylpyrrolidone (PVP) will be used to conjugate Ag NPs with curcumin (Ag-PVP-C) to make it bioavailable without harming its medicinal activity. Then the optical, vibrational, and morphological properties of the synthesized metallic NPs will be considered. Moreover, the modification of these properties with conjugation of therapeutic agent curcumin will be 
studied. Finally, a critical evaluation with respect to cellular (MDA-MB-231 breast cancer cells) uptake of the PVPcapped Ag NPs/Ag-PVP-CNPs (curcumin conjugated PVP-capped Ag NPs) will be carried out through fluorescence imaging data.

\section{Experimental details}

\subsection{Synthesis of PVP capped Ag nanoparticles (Ag NPs)}

Silver nitrate $\left(\mathrm{AgNO}_{3}\right)$ has been used as a metal precursor and borohydride $\left(\mathrm{NaBH}_{4}\right)$ as reducing agent for the preparation of Ag NPs. At first, $1 \%$ PVP (MW 40000) solution was prepared by dissolving $0.1 \mathrm{~g}$ of PVP in $10 \mathrm{~mL}$ distilled water and stirred it for $30 \mathrm{~min}$. Then $10 \mathrm{~mL}$ volume of $1 \mathrm{mM}$ stock solution of $\mathrm{AgNO}_{3}$ was prepared by dissolving $0.00169 \mathrm{~g}$ silver salt in $10 \mathrm{~mL}$ distilled water. From the stock solution, $10 \mathrm{~mL}$ of $0.4 \mathrm{mM}$ solution was prepared and added to the PVP solution. After 15 minutes of stirring, $2 \mathrm{~mL}$ of $0.2 \mathrm{mM}$ sodium borohydride $\left(\mathrm{NaBH}_{4}\right)$, freshly prepared in ice cold water, was added to the above mixture in a drop wise manner. With the addition of $\mathrm{NaBH}_{4}$, the color of the solution turned to light yellow indicating the formation of the Ag NPs by reduction of $\mathrm{Ag}^{2+}$ to $\mathrm{Ag}^{0}$. The solution was then cooled to room temperature.

\subsection{Synthesis of curcumin conjugated PVP capped Ag nanoparticles (Ag-PVP-C NPs)}

Conjugation of curcumin with PVP-capped Ag NPs was carried out by dissolving $30 \mathrm{mg}$ of curcumin in $10 \mathrm{~mL}$ of acetone. Acetone was used for as the solubility of curcumin is highest in acetone as compared to other commonly used organic solvents, such as methyl ethyl ketone, ethyl acetate, methanol, ethanol, 1,2-dichloroethane, isopropanol, ether etc.

$5 \mathrm{~mL}$ curcumin solution was then added to $10 \mathrm{~mL}$ solution of the as synthesized PVP-capped Ag NPs, under stirring at $100{ }^{\circ} \mathrm{C}$ temperature. The reaction was allowed to continue for up to $2 \mathrm{~h}$ to get curcumin conjugated-PVP capped Ag NPs (Ag-PVP-C).

\subsection{Characterization techniques}

The structural characterization of the Ag NPs and Ag-PVP-C NPs complex was performed by using a highresolution transmission electron microscopy (HRTEM) (Model: JEM-2100, JEOL, USA) at NEHU, Shillong, India, working at an accelerating voltage of $200 \mathrm{kV}$. The optical absorption study was performed by the UV-Visible absorption spectroscopy (UV 2450, Shimadzu Corporation). The zeta potential was measured using a Malvern Zetasizer (Model Nano ZS, NSW, Australia). Furthermore, IR-active vibrational features were assessed through Fourier transform infrared (FT-IR) (Nicolet model Impact-410) studies. Ag NPs and Ag-PVP-C NPs complexes were used as cancer cell probes by using a fluorescent microscope (Model: Leica DM300, USA) equipped with a cooled color CCD camera (Model DP71).

\section{Results and discussions}

We discuss below the analysis of PVP capped Ag NPs (Ag NPs) and curcumin conjugated-PVP capped Ag NPs (Ag-PVP-C) characterized by different techniques.

\subsection{Morphological analysis through transmission electron microscopy studies}

High resolution transmission electron microscopy (HRTEM) was used to study themorphological behavior of the as-synthesized PVP capped Ag NPs and curcumin conjugated PVP-capped Ag NPs. The HRTEM micrographs of the PVP capped Ag NPs are shown in Fig. 1(a). The average size (diameter) of the NPs was found to be $\sim 2 \mathrm{~nm}$ with nearly spherically symmetric structure. Further, good crystallinity of the sample could be predicted from the clear lattice fringes (upper left inset of Fig. 1(a)). The typical interplanar spacing $(d)$ was estimated to be $\sim 0.2 \mathrm{~nm}$ which is close to the value $(0.23 \mathrm{~nm})$ reported in an earlier work [12]. The perfect periodicity of lattice atoms was mostly witnessed in the central region (approx. at a distance within $\sim 0.7 \mathrm{~nm}$ from the center) while missing atoms and planes are generally observable close to the surface of the Ag nanoparticle. The orientation of the planes of the upper right side of a particle changes the orientation with regard the lower side planes ((lower left inset of Fig. 1(a)). This may suggest the existence of some edge dislocation occurring as a result of undeveloped lattice planes at the nanoparticle surface.

The HRTEM image of curcumin conjugated-PVP capped Ag NPs (Ag-PVP-C NPs) is shown in Fig. 1(b). The average size of the Ag-PVP-C NPs are found to be $\sim 4.25 \mathrm{~nm}$ in diameter. It can be observed that Ag NPs are surrounded by PVP. Similar behavior has also been observed for PVP-capped Au NPs conjugated to curcumin [13]. 

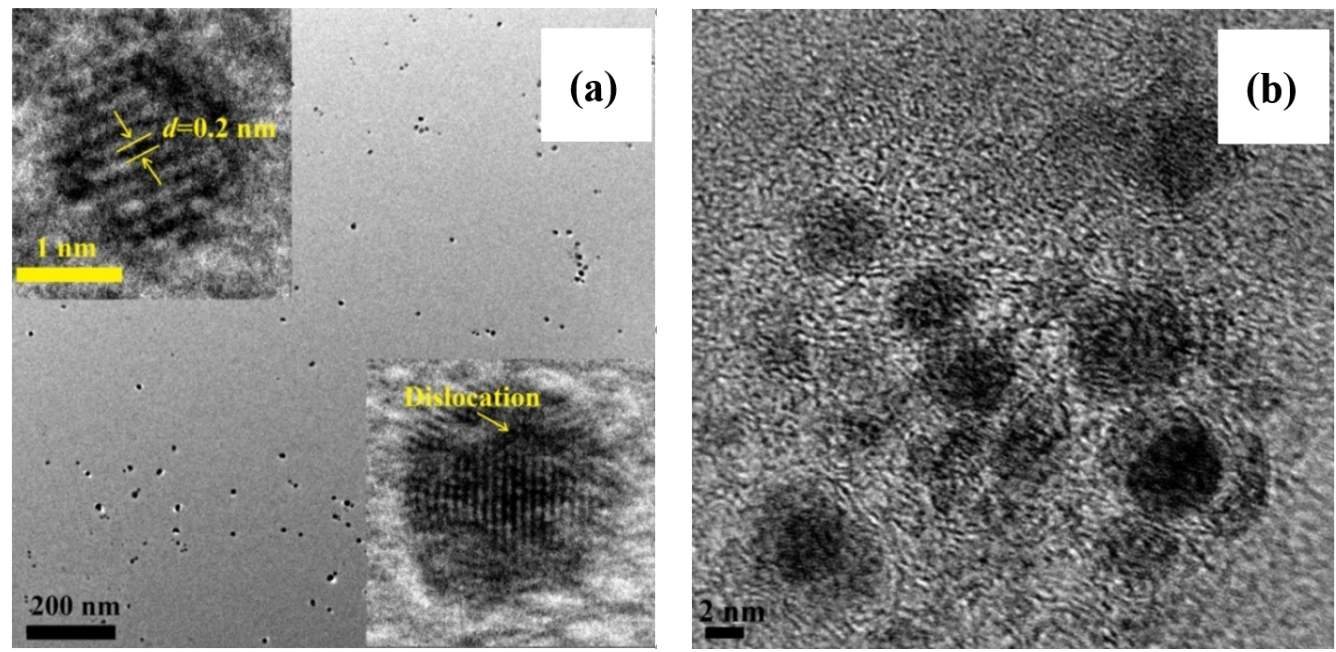

FIG. 1. HRTEM image of (a) PVP capped Ag NPs. The enlarged, isolated NPs are shown as upper left and lower right insets with indicating interplanar spacing and dislocation; respectively.

(b) HRTEM image of curcumin conjugated-PVP capped Ag NPs (Ag-PVP-C)

\subsection{UV-Vis spectra of PVP capped Ag NPs, curcumin (C) and Ag-PVP-C NPs}

The UV-Vis absorption spectra of PVP capped Ag NPs, curcumin and Ag-PVP-C NPs are depicted in Fig. 2. As can be found, Ag NPs shows SPR peak at $\sim 400 \mathrm{~nm}$ arising from the collective oscillation of free conduction electrons induced by an interacting electromagnetic field (Fig. 2(a)).

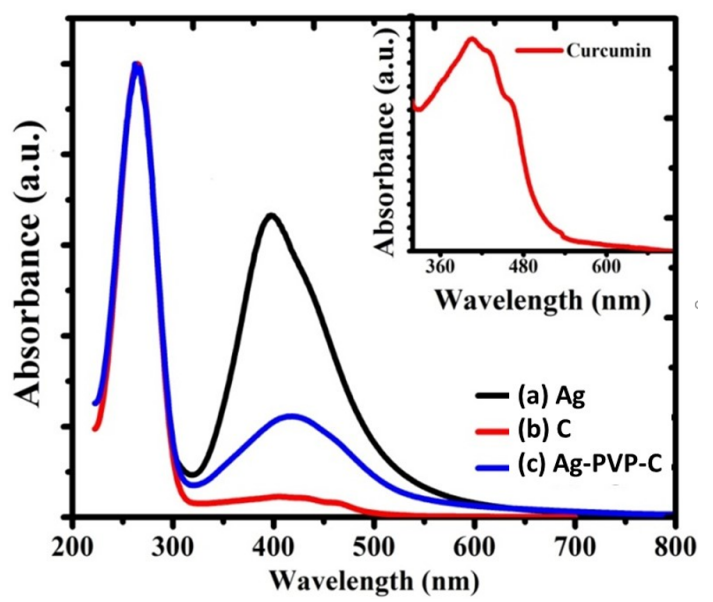

FIG. 2. Optical absorption of (a) PVP capped Ag NPs (Ag); (b) Curcumin(C); (c) Curcumin conjugated-PVP capped Ag NPs (Ag-PVP-C)

Curcumin generally shows a strong and intense absorption band in the ranges of $350-480 \mathrm{~nm}$ and $200-280 \mathrm{~nm}$. In our case (Fig. 2(b)), the absorption spectrum in the region of $350-480 \mathrm{~nm}$ is very broad, having a number of absorption bands. The presence of more than one shoulder i.e. at $\sim 406,429$ and $462 \mathrm{~nm}$, (shown in enlarged in the inset of the figure) indicates the possible presence of more than one isomeric form in the ground state of curcumin $[14,15]$. Moreover, is to be noted that the peak observed at $\sim 429 \mathrm{~nm}$ is a signature of the basic diarylheptanoid chromophore group of curcumin $[6,16]$, the main therapeutic group of this molecule. One characteristic absorption band of curcumin, which correspond to the transfer of $\pi-\pi^{*}$ electrons in the benzene ring, is noticed at $\sim 263 \mathrm{~nm}$.

The UV-Vis spectra of curcumin conjugated PVP-capped Ag NPs (Ag-PVP-C)(Fig. 2(c)) clearly shows two distinct peaks at $\sim 263$ and $418 \mathrm{~nm}$. The peak observed at $\sim 418 \mathrm{~nm}$ is red shifted by $18 \mathrm{~nm}$ from original SPR peak at $400 \mathrm{~nm}$ as observed in curcumin free PVP capped Ag NPs. Whereas, this peak is blue shifted by $\sim 11 \mathrm{~nm}$ from the curcumin absorption peak at $\sim 429 \mathrm{~nm}$ related to the diarylheptanoid chromophore group. The presence of this peak 
at Ag-PVP-C complex demonstrates that curcumin retains its diarylheptanoid chromophore group while conjugating to PVP capped Ag NPs, which is much needed in antimicrobial applications $[6,17]$.

\subsection{Molecular vibrations in PVP capped Ag NPs, curcumin (C) and Ag-PVP-C NPs}

Figure 3 shows the FTIR spectra of PVP capped Ag NPs, curcumin (C) and curcumin conjugated-PVP capped Ag NPs (Ag-PVP-C).

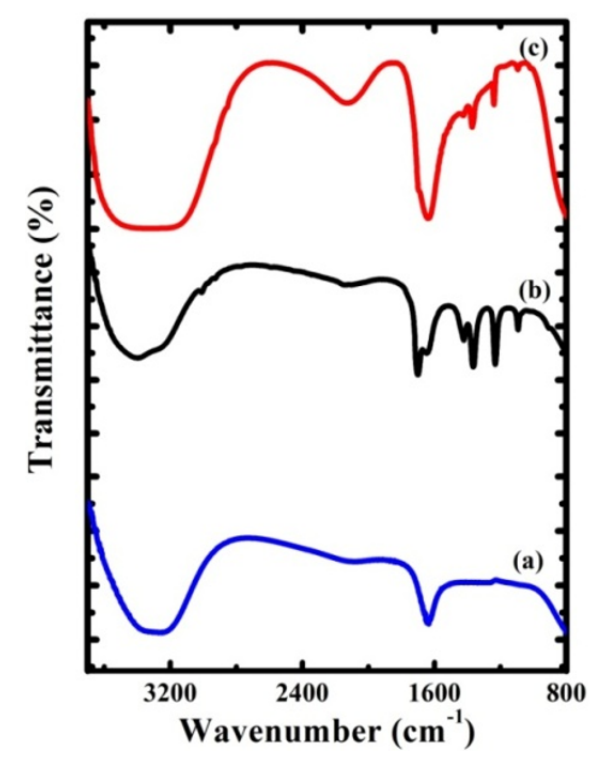

FIG. 3. FTIR spectra of (a) PVP capped Ag NPs; (b) Curcumin (C); (c) Curcumin conjugated PVPcapped Ag NPs (Ag-PVP-C)

Figure 3(a) shows the FTIR spectra of the PVP-capped Ag NPs. A broad band centered around $3412 \mathrm{~cm}^{-1}$ attributed to the stretching vibration of $-\mathrm{OH}$ group. The absorption bandof the $\mathrm{C}=\mathrm{O}$ bond at $1663 \mathrm{~cm}^{-1}$ for pure $\mathrm{PVP}$ was shifted to $1643 \mathrm{~cm}^{-1}$ for Ag NPs capped by PVP. This decrease in wavenumber for the $\mathrm{C}=\mathrm{O}$ absorption may result from bond weakening via partial donation of oxygen loan pair electrons of PVP to the vacant orbitals of the silver [18]. Whereas, the wavenumber observed at $\sim 1270 \mathrm{~cm}^{-1}$ was due to bond vibrations of the $\mathrm{N}=\mathrm{H}-\mathrm{O}$ complex [19]. Thus, the FTIR spectra reveal the molecular interaction between Ag and PVP chain.

To determine the specific sites of interaction between PVP-capped Ag NPs and curcumin, FTIR spectra of both curcumin (Fig. 3(b)) and curcumin conjugated-PVP capped Ag NPs (Ag-PVP-C) (Fig. 3(c)) have been studied. The FTIR bands for stretching vibration of $-\mathrm{OH}$ group in curcumin is observed at $3398 \mathrm{~cm}^{-1}$. This $-\mathrm{OH}$ vibration absorption (at $\sim 3321 \mathrm{~cm}^{-1}$ ) observed for curcumin conjugated PVP-capped Ag NP is not sharp as obtained for curcumin. This may be due to the hydrogen-bond intermolecular interaction between $\mathrm{O}-\mathrm{H}$ of curcumin and $\mathrm{C}=\mathrm{O}$ of $\mathrm{PVP}$ around Ag NP [20].

The FTIR band due to the in-plane bending of two phenolic and one enolic hydroxyl groups of the curcumin are observed at 1360 (phenolic) 1227 (phenolic) and $962 \mathrm{~cm}^{-1}$ (enolic). The absence of a band of at $962 \mathrm{~cm}^{-1}$ (due to enolic group of curcumin) for the Ag-PVP-C compound may suggest the interaction of Ag NPs through this site also [21]. Generally, metal coordination of curcumin occurs through the enolic group, where the enolic proton is replaced by the metal ion and the $o$-methoxy phenolic moiety remains intact in the complexes [22].

The band at $1428 \mathrm{~cm}^{-1}$ observed in curcumin corresponds to the olefinic in-plane bending vibrations of the heptadiene chain of curcumin. Similarly, the absorption at, $1091 \mathrm{~cm}^{-1}$ is due to aromatic C-O-C vibration of curcumin. These bands at 1428 and $1091 \mathrm{~cm}^{-1}$ shown by curcumin as stated above were shifted to 1424 and $1178 \mathrm{~cm}^{-1}$; respectively on conjugation of curcumin to PVP capped Ag NPs. This indicates the presence of intact curucmin moiety in the Ag-PVP-C complex [21]. The shifting of aromatic C-O-C vibration in Ag-PVP-C NP signifies the symmetry change related to benzene rings of curcumin [17]. The presence of basic diarylheptanoid group is confirmed from the FTIR bandat $1424 \mathrm{~cm}^{-1}$ in Ag-PVP-C NPs. This is the chromophore group of curcumin which is required in the antimicrobial application. Thus, FTIR spectra confirm that curcumin retains its diarylheptanoid chromophore group while conjugating to PVP capped Ag NPs and hence preserving its therapeutic properties. 


\subsection{Measurement of zeta potential}

The stability of a colloidal system could be predicted from the value of the zeta potential. An increased value of zeta potential $(+v e$ or $-v e$ ) signifies the enhancement of repulsive behavior between the particles and thus results a more stable colloidal dispersion. The respective values of zeta potential of PVP-capped Ag NPs (Ag NPs) and curcumin conjugated PVP-capped Ag NPs (Ag-PVP-C) were observed to be -16.5 and $-33.7 \mathrm{mV}$. The larger value of zeta potential in Ag-PVP-C clearly indicates the efficient conjugation of curcumin to PVP capped Ag NPs while simultaneously enhancing its stability in the colloidal solution.

\subsection{PVP capped Ag NPs (Ag NPs) and curcumin conjugated-PVP capped Ag NPs (Ag-PVP-C NPs) as cancer cell probe}

Imaging snapshots of MDA-MB-231 cancer cells treated with PVP capped Ag NPs and curcumin conjugated PVP-capped Ag NPs are shown in Fig. 4(a) and (b), respectively. The snapshots suggest high biocompatibility and fluorescent behavior of both PVP capped Ag NPs and curcumin conjugated PVP capped Ag NPs in the cancer cellular environment.

Using image J-1.46r software ${ }^{\circledR}$ the quantitative analysis of the fluorescence of the NPs was predicted presuming complete localization of the NPs inside the cell as discussed in previous reports [23,24]. Fig. 4(c,d) highlight the areas of interest (A, B, C, D etc.) and the selected background areas (region without fluorescence; bk1, bk2, bk3 etc.) of fluorescent images marked with encircled regions. The corrected total counts of fluorescence (CTCF) intensity values were calculated using the relation:

$$
\mathrm{CTCF}=\text { Integrated Density }-(\text { Area } \times \text { mean fluorescent of background setting }),
$$

here, the 'integrated density' (IntDen) of a fluorescent image is the sum of the values of the pixels in the selected regions. Fig. 4(e) signifies a comparative view on representative histograms of the average CTCF and average IntDen obtained for different NPs system. It predicts biocompatibility and fluorescent behavior of the NPs on being used for the treatment of cancer cells. Moreover, the fluorescence intensity indicates the level of the internalization of NPs by the cells. It could be observed that, the fluorescence efficiency (CTCF) of curcumin conjugated-PVP capped Ag NPs in the cancer cellular medium is $\sim 2.37$ times more than that the value obtained for curcuminfree PVP-capped Ag NPs. Thus, the cellular uptake and bioimaging aspects of metallic Ag NPs were significantly affected by the conjugation with the therapeutic agent curcumin. Similar response for the curcumin conjugated NPs is also reported earlier [25]. Though the surface charge/zeta potential of NPs significantly impact the cellular uptake mechanism, there are different opinions regarding uptake rate of cationic and anionic NPs by the cells [26-30]. Moreover, there may be direct permeation of the NPs in to the cell irrespective of the surface charge of the NPs [31].

Fröhlich, in a review article [29], discusses the role of surface charge of the NPs on cytotoxicity, cellular uptake, and their localization in the intracellular region. It was concluded that nonphagocytic cells have the ability to uptake cationic NPs to a higher extent, while phagocytic cells favor anionic NPs. Apart from the surface charge, there are different factors such as size, shape, type of material, charge density, surface hydrophobicity, concentration and stabilizing agents that influence the cellular uptake process of a NP.

MDA-MB-231 breast cancer cells have the potential to function as phagocytes. They exhibit phagocytic activity (engulfing and digesting) on the normal cells $[32,33]$. Cellular uptake of PVP-capped anionic Ag NPs occur in the MDA-MB-231 breast cancer cells in endosomes and then in the amphisomes (of the MDA-MB-231 breast cancer cells) [34]. However, there is aggregation/degradation of the PVP-capped Ag NPs inside the cancer cells. In the present case, we can expect preferential internalization of the curcumin conjugated PVP-capped Ag NPs (zeta potential $-33.7 \mathrm{mV}$ ) by phagocytic MDA-MB-231 breast cancer cells. As obtained from zeta potential curcumin conjugated PVP-capped Ag NPs show better colloidal stability preventing their aggregation and hence the degradation. Moreover, PVP-capped Ag NPs may increase the photostability of curcumin and hence reduce photobleaching resulting higher fluorescence of the curcumin conjugated PVP-capped Ag NPs inside the cells [35].

An electrostatic repulsive force between anionic NPs and the negatively charged cell membrane can hinder the efficiency of NPs binding to the cell. This electrostatic repulsion can be suppressed by changing the capping/conjugated material and the size of the NPs [36]. In this context, detailed of both PVP-capped Ag NPs and curcumin conjugated PVP-capped Ag NPs which are anionic particles, are needed to determine the existence or extent of influence of electrostatic repulsive behavior on the cellular uptake rates in the breast cancer cells considered in our study. However, at present, a comprehensive assessment of this topic is beyond the present scope [37]. Though anionic NPs are characterized by limited interaction ability, they are attractive for biomedical applications due to very low cytotoxic effects as compared to the cationic ones. There are various reports which describe successful internalization of anionic NPs to cells $[36,38-40]$. 

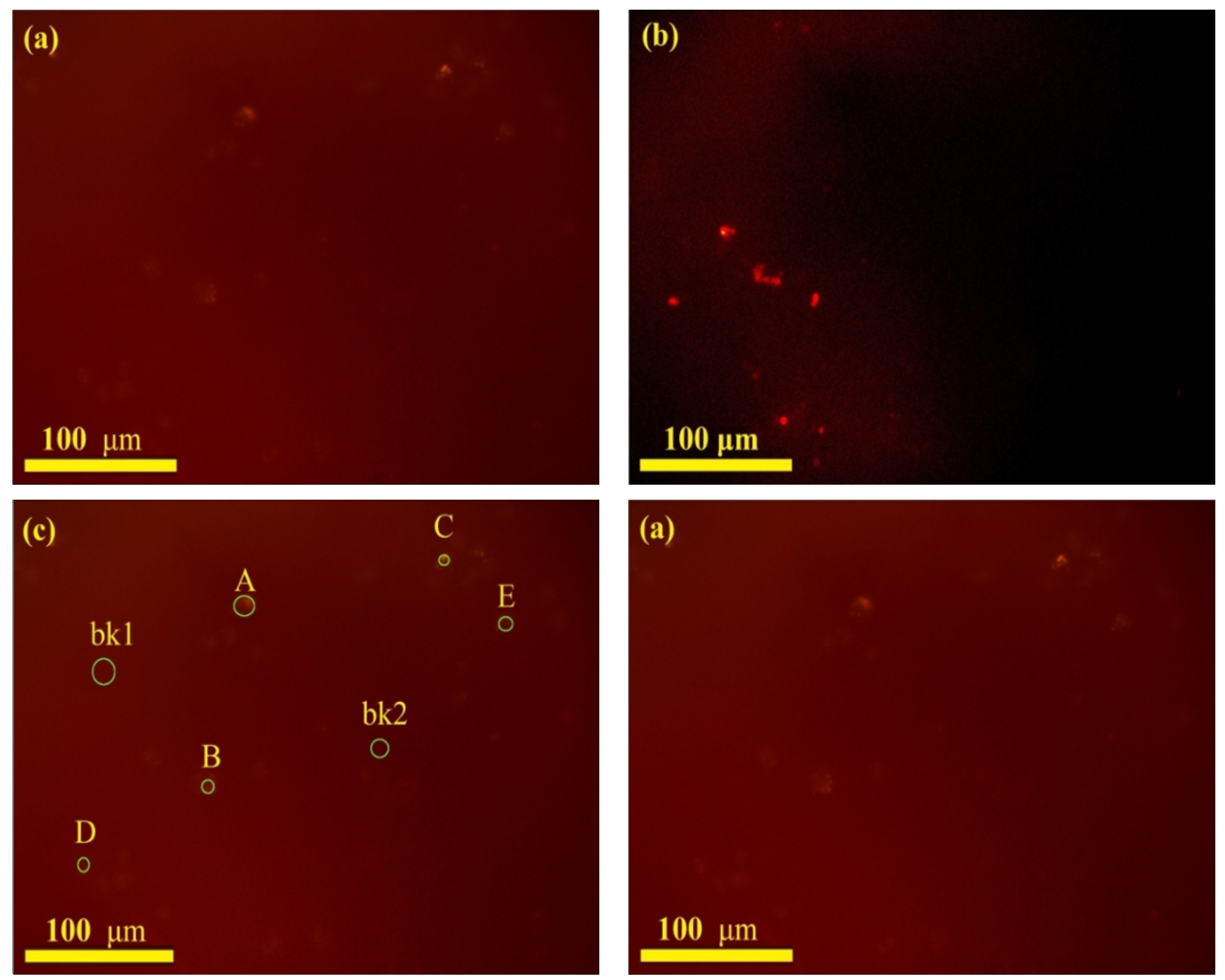

(e)

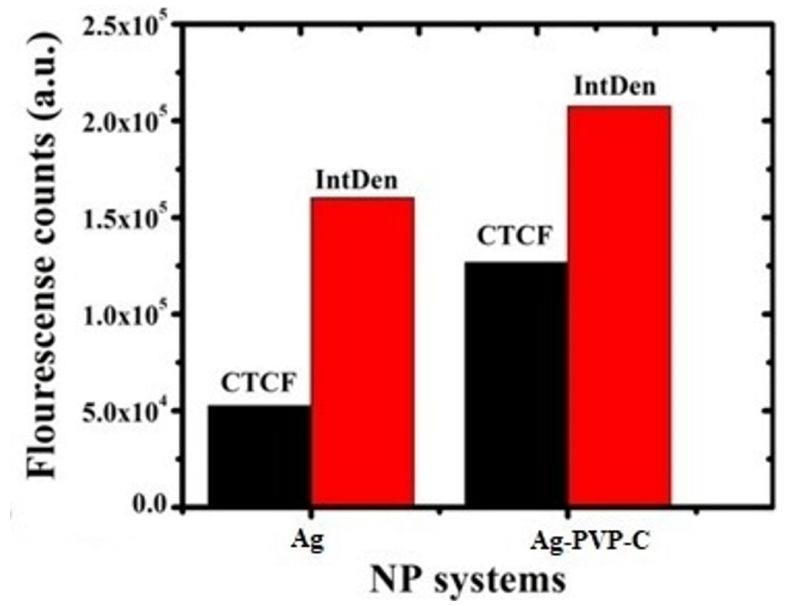

FIG. 4. Fluorescent imaging (using violate filter) of cancer cells with (a) Ag NPs (b) Ag-PVP-C complex. Figure (c), (d) show selected fluorescent and background areas of fluorescent images respectively $\left(\lambda_{e x} \sim 300 \mathrm{~nm}\right)$. Histograms representing the average value of IntDen and CTCF of different types of NP systems is shown in Figure (e) 


\section{Conclusion}

PVP-capped silver nanoparticles were synthesized and characterized for structural, optical and vibrational properties. The influence of curcumin on the aforementioned properties have been studied by conjugating curcumin to PVP-capped Ag NPs. Curcumin was found to be water soluble and retained its therapeutic properties upon conjugation with PVP capped Ag NPs. While used as a cancer cell imaging probe, curcumin conjugated PVP-capped Ag NPs showed higher fluorescence response as compared to the curcumin free ones. The production of water soluble and biocompatible curcumin expands the clinical range of this efficacious agent without hampering the environment and human health. In addition, the curcumin conjugated PVP-capped Ag NPs exhibited fluorescent properties, which is believed to cater to a broad spectrum of diverse applications in biological and pharmacological activities such as bio-imaging, targeted drug delivery, biosensing, examination of intracellular processes etc.

\section{Acknowledgements}

The author R. Sarmais thankful to the Department of Biotechnology (DBT), Ministry of Science \& Technology, New Delhi for financial support under DBT-RA scheme Department of Biotechnology, government of India, New Delhi (Project No. GAP0740). The authors are also thankful to the Material Science Division, CSIR-NEIST, Jorhat for providing nanoparticle synthetic lab facility, UV-Vis and Zeta potential measurement, and Director, CSIR-NEIST, Jorhat for providing the necessary facilities in the institute, and Dr. Manash Jyoti Kashyap, for his extensive help in editing the article. The authors gratefully acknowledge Analytical Chemistry Group, CSIR-NEIST for FTIR analysis and SAIF-NEHU, Shillong for HR-TEM measurements.

\section{References}

[1] Anand P., et al. Bioavailability of curcumin: problems and promises. Molecular pharmaceutics, 2007, 4 (6), P. $807-818$.

[2] Senft C., et al. The nontoxic natural compound Curcumin exerts anti-proliferative, anti-migratory, and anti-invasive properties against malignant gliomas. BMC cancer, 2010, 10 (1), P. 491.

[3] Lao C.D., et al. Dose escalation of a curcuminoid formulation. BMC complementary and alternative medicine, 2006,6 (1), P. 10.

[4] Qureshi S., Shah A., Ageel A. Toxicity studies on Alpinia galanga and Curcuma longa. Planta medica, 1992, 58 (2), P. $124-127$.

[5] Daniel S., et al. Through metal binding, curcumin protects against lead-and cadmium-induced lipid peroxidation in rat brain homogenates and against lead-induced tissue damage in rat brain. Journal of inorganic biochemistry, 2004, 98 (2), P. $266-275$.

[6] Gangwar R.K., et al. Conjugation of curcumin with PVP capped gold nanoparticles for improving bioavailability. Materials Science and Engineering C, 2012, 32 (8), P. 2659-2663.

[7] Gopinath P., et al. Implications of silver nanoparticle induced cell apoptosis for in vitro gene therapy. Nanotechnology, 2008,19 (7), 075104.

[8] Wu W., et al. Smart core- shell hybrid nanogels with Ag nanoparticle core for cancer cell imaging and gel shell for pH-regulated drug delivery. Chemistry of Materials, 2010, 22 (6), P. 1966-1976.

[9] Shahverdi A.R., et al. Synthesis and effect of silver nanoparticles on the antibacterial activity of different antibiotics against Staphylococcus aureus and Escherichia coli. Nanomedicine: Nanotechnology, Biology and Medicine, 2007, 3 (2), P. 168-171.

[10] Kravets V., et al. Imaging of biological cells using luminescent silver nanoparticles. Nanoscale research letters, 2016,11 (1), P. 30.

[11] Tran Q.H., Le A.-T. Silver nanoparticles: synthesis, properties, toxicology, applications and perspectives. Advances in Natural Sciences: Nanoscience and Nanotechnology, 2013, 4 (3), 033001.

[12] Jyoti K., Baunthiyal M., Singh A. Characterization of silver nanoparticles synthesized using Urtica dioica Linn. leaves and their synergistic effects with antibiotics. Journal of Radiation Research and Applied Sciences, 2016, 9 (3), P. 217-227.

[13] Gangwar R.K., et al. Conjugation of curcumin with PVP capped gold nanoparticles for improving bioavailability. Materials Science and Engineering C, 2012, 32 (8), P. 2659-2663.

[14] Patra D., Barakat C. Synchronous fluorescence spectroscopic study of solvatochromic curcumin dye. Spectrochimica Acta Part A: Molecular and Biomolecular Spectroscopy, 2011, 79 (5), P. 1034-1041.

[15] Masek A., Chrzescijanska E., Zaborski M. Characteristics of curcumin using cyclic voltammetry, UV-vis, fluorescence and thermogravimetric analysis. Electrochimica Acta, 2013, 107, P. 441-447.

[16] Masuda T., et al. Chemical studies on antioxidant mechanism of curcuminoid: analysis of radical reaction products from curcumin. Journal of agricultural and food chemistry, 1999, 47 (1), P. 71-77.

[17] Hatamie S., et al. Complexes of cobalt nanoparticles and polyfunctional curcumin as antimicrobial agents. Materials Science and Engineering C, 2012, 32 (2), P. 92-97.

[18] Ajitha B., et al. Role of capping agents in controlling silver nanoparticles size, antibacterial activity and potential application as optical hydrogen peroxide sensor. RSC Advances, 2016, 6 (42), P. 36171-36179.

[19] Zhang Z., Zhao B., Hu L. PVP protective mechanism of ultrafine silver powder synthesized by chemical reduction processes. Journal of Solid State Chemistry, 1996, 121 (1), P. 105-110.

[20] Machmudah S., et al. Formation of Fine Particles from Curcumin/PVP by the Supercritical Antisolvent Process with a Coaxial Nozzle. ACS omega, 2020, 5 (12), P. 6705-6714.

[21] Sindhu K., et al. Curcumin conjugated gold nanoparticle synthesis and its biocompatibility. RSC Advances, 2014, 4 (4), P. 1808-1818.

[22] Priyadarsini K. The Chemistry of Curcumin: From Extraction to Therapeutic Agent. Molecules, 2014,19 (12), P. 20091.

[23] Sarma R., Mohanta D. Luminescence and bio-imaging response of thio-glycolic acid (TGA) and sodium dodecyl sulfate (SDS)-coated fluorescent cadmium selenide quantum dots. Journal of Luminescence, 2015, 161, P. 395-402.

[24] Burgess A., et al. Loss of human Greatwall results in G2 arrest and multiple mitotic defects due to deregulation of the cyclin B-Cdc2/PP2A balance. Proceedings of the National Academy of Sciences, 2010, 107 (28), P. 12564-12569. 
[25] Nguyen H.N., et al. Curcumin as fluorescent probe for directly monitoring in vitro uptake of curcumin combined paclitaxel loaded PLA-TPGS nanoparticles. Advances in Natural Sciences: Nanoscience and Nanotechnology, 2016, 7 (2), P. 025001.

[26] Patil S., et al. Protein adsorption and cellular uptake of cerium oxide nanoparticles as a function of zeta potential. Biomaterials, 2007, 28 (31), P. 4600-4607.

[27] Zhao M.-X., Zeng E.-Z. Application of functional quantum dot nanoparticles as fluorescence probes in cell labeling and tumor diagnostic imaging. Nanoscale research letters, 2015, 10 (1), P. 171.

[28] Foroozandeh P., Aziz A.A. Insight into Cellular Uptake and Intracellular Trafficking of Nanoparticles. Nanoscale research letters, 2018, 13 (1), P. 339.

[29] Fröhlich E. The role of surface charge in cellular uptake and cytotoxicity of medical nanoparticles. International journal of nanomedicine, 2012, 7, P. 5577-5591.

[30] Jeon S., et al. Surface Charge-Dependent Cellular Uptake of Polystyrene Nanoparticles. Nanomaterials (Basel, Switzerland), 2018, 8 (12), P. 1028.

[31] Nakamura H., Watano S. Direct Permeation of Nanoparticles Across Cell Membrane: A Review. Powder and Particle, $2018,35$.

[32] Ivers L.P., et al. Dynamic and influential interaction of cancer cells with normal epithelial cells in 3D culture. Cancer Cell International, 2014, 14 (1), P. 108.

[33] Monks J., et al. Epithelial cells as phagocytes: apoptotic epithelial cells are engulfed by mammary alveolar epithelial cells and repress inflammatory mediator release. Cell Death \& Differentiation, 2005, 12 (2), P. 107-114.

[34] Swanner J., et al. Silver nanoparticles selectively treat triple-negative breast cancer cells without affecting non-malignant breast epithelial cells in vitro and in vivo. FASEB BioAdvances, 2019, 1 (10), P. 639-660.

[35] Abdellah A.M., et al. Green synthesis and biological activity of silver-curcumin nanoconjugates. Future medicinal chemistry, 2018, 10 (22), P. 2577-2588.

[36] Mendozza M., et al. Nanoparticles and organized lipid assemblies: from interaction to design of hybrid soft devices. Soft Matter, 2019, 15 (44).

[37] Doherty G.J., McMahon H.T. Mechanisms of Endocytosis. Annual Review of Biochemistry, 2009, 78 (1), P. 857-902.

[38] Zhang X., et al. Effects of surface charges of gold nanoclusters on long-term in vivo biodistribution, toxicity, and cancer radiation therapy. International journal of nanomedicine, 2016, 11, P. 3475-3485.

[39] Alexis F., et al. Factors Affecting the Clearance and Biodistribution of Polymeric Nanoparticles. Molecular Pharmaceutics, 2008, 5 (4), P. 505-515.

[40] Bodewein L., et al. Differences in toxicity of anionic and cationic PAMAM and PPI dendrimers in zebrafish embryos and cancer cell lines. Toxicology and Applied Pharmacology, 2016, 305, P. 83-92. 